\title{
Long-term follow up of patients with multiple gastric carcinoids associated with type $A$ gastritis
}

\author{
Osamu Hosokawa ${ }^{1}$, Yasuharu Kaizaki ${ }^{2}$, Masakazu Hattori ${ }^{1}$, Kenji Douden ${ }^{1}$, Hiroyuki Hayashi ${ }^{1}$, \\ Minoru Morishita ${ }^{1}$, and Kouji OHTA ${ }^{1}$ \\ ${ }^{1}$ Department of Surgery, Fukui Prefectural Hospital, 2-8-1 Yotsui, Fukui 910-8526, Japan \\ ${ }^{2}$ Department of Pathology, Fukui Prefectural Hospital, Fukui, Japan
}

\begin{abstract}
Little is known about the natural course of multiple gastric carcinoids associated with type A gastritis. Between 1993 and 2003, we enrolled eight patients, diagnosed as having multiple gastric carcinoids associated with type $\mathbf{A}$ gastritis, in a followup program without surgical resection. In these patients, endoscopy showed multiple small polyps on the gastric body, with nonantral atrophic gastritis. Histologically, biopsy specimens obtained from the polyps revealed carcinoid tumors. The serum gastrin level was found to be very high in all patients, and testing for anti-parietal cell antibody was positive in seven. The mean follow-up was 5.8 years (range, 1.5-10.8 years). The levels of serum gastrin increased in all patients, but, endoscopically, the carcinoid tumors did not change in size. Neither hepatic nor lymphatic metastasis was detected on abdominal computed tomography (CT). These patients were free of the development or metastasis of carcinoids, in spite of their continuous hypergastrinemia. It was concluded that multiple gastric carcinoids associated with type A gastritis may be indolent.
\end{abstract}

Key words Gastric carcinoid - Type A gastritis - Hypergastrinemia $\cdot$ Endoscopic follow up

\section{Introduction}

Type A gastritis is associated with an autoimmune disease and involves mainly the fundus and body of the stomach [1]. It is generally seen in elderly patients, in whom severe atrophy of the mucosa develops. Those affected have antibodies against gastric parietal cells and intrinsic factor, leading to failure of absorption of dietary vitamin B12, followed by pernicious anemia. Gastric carcinoid tumors are often found in patients diagnosed as having type A gastritis. In patients with type A gastritis, the development of carcinoids and the

Offprint requests to: O. Hosokawa

Received: April 13, 2004 / Accepted: September 17, 2004 widespread hyperplasia of enterochromaffin-like cells are related to atrophic changes of the fundic mucosa and the trophic action of subsequently raised serum gastrin levels [2].

In Japan, total or subtotal gastrectomy has been performed for patients with gastric carcinoids associated with type A gastritis [3]. In 1991, we also carried out total resection of the stomach in a patient initially diagnosed as having this disease [4]. However, histological examination of the resected specimens suggested that the disease would be indolent, and that more conservative treatment should be used [5]. Subsequently, we have recommended that patients with multiple gastric carcinoids associated with type A gastritis should not receive surgical resection, but should have endoscopic follow up. In such patients enrolled in our long-term follow-up program, the disease has not progressed morphologically.

\section{Case reports}

Ten patients with multiple carcinoids associated with type A gastritis have been admitted to our hospital. The first patient was operated on, with a total gastrectomy, but histological examination of the resected specimens revealed that the tumor would be indolent [4]. Subsequently we recommended more conservative treatment for patients affected by such disease. The second patient refused such treatment, but the third agreed.

Between 1993 and 2003, eight patients (three men and five women) were enrolled in our follow-up program. The mean age of the patients was 57.4 years (range, 46-69 years) at diagnosis (Table 1). Endoscopic examinations showed multiple small polyps on the gastric body (Fig. 1A), with non-antral atrophic gastritis. The polyps measured between 1 and $15 \mathrm{~mm}$, and were covered by intact mucosa identical to the surrounding tissue. Histological examination of biopsy specimens 


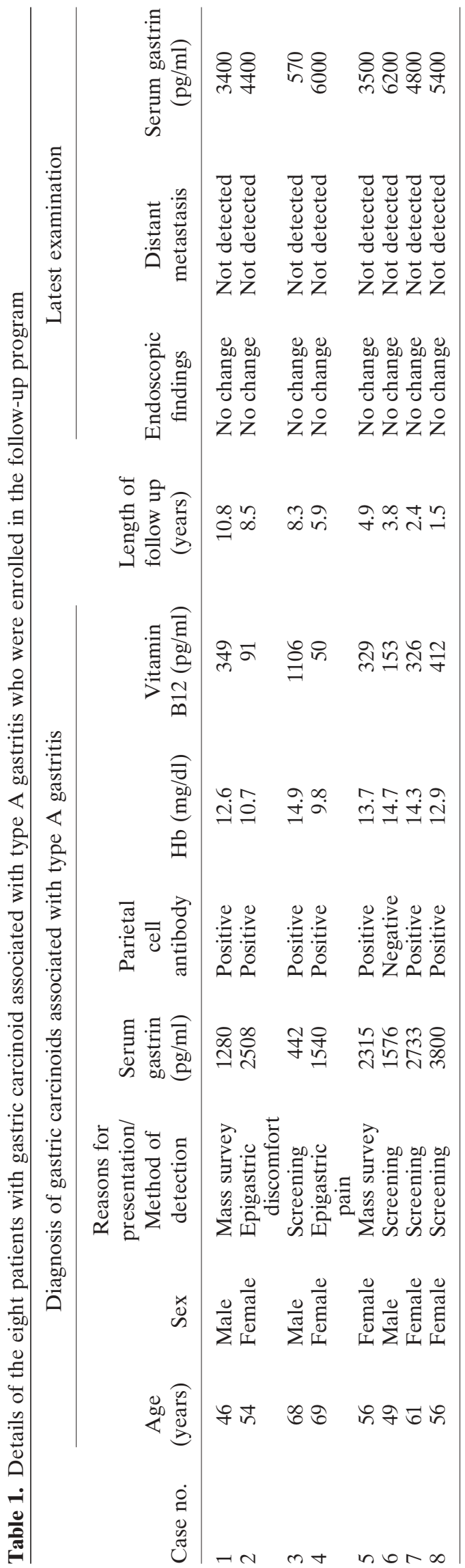

obtained from the polyps revealed carcinoid tumors. The mean plasma gastrin level of these patients was high, at $2024 \mathrm{pg} / \mathrm{ml}$ (range, $442-3800 \mathrm{pg} / \mathrm{ml}$ ); however, concentrations of somatostatin, histamine, and serotonin were not elevated. Moreover, serum pepsinogen I and II levels were very low, and testing for anti-parietal cell antibody was positive in seven patients. Pernicious anemia affected two patients who were examined for abdominal complaints.

\section{Follow-up program}

The patients and their families were informed about the disease, and endoscopic follow up was recommended according to the ethics regulations at our hospital. The patients and families agreed with our proposition, and we started the follow-up program, which consisted of endoscopic examinations at 6-month intervals and computed tomography (CT) scans and blood tests at 12month intervals.

Follow-up endoscopy showed that the polyps had the same size as on the initial picture (Fig. 2B), and histological examination of recent biopsy specimens of the polyps revealed residual carcinoid tumor in the mucosal layer (Fig. 3A). CT examination findings were normal and serum gastrin levels had increased in all patients at the end of follow up (Table 1).

During this long-term follow up, these eight patients have been free of the development or metastasis of carcinoids. Multiple gastric carcinoids associated with type A gastritis may be indolent, in spite of the patients having continuous hypergastrinemia.

\section{Discussion}

In Japan, total gastrectomy with lymph node dissection has been the prevailing operative procedure for the treatment of multiple gastric carcinoids associated with type A gastritis. In a review of the Japanese literature, Yamamoto et al. [3] reported that, between 1975 and 1995, 33 of 35 patients affected with such disease were operated on with a total or subtotal gastrectomy. The purpose of this procedure was to completely resect the fundic gland area which could be the origin of the carcinoids and endocrine cell micronests [6].

Eckhauser et al. [7] reported that antrectomy and regional lymphadenectomy was performed in two patients with multicentric, argyrophil gastric carcinoids. The tumors were locally invasive, with penetration through the submucosa. One patient had regional lymph node involvement, and one had an isolated hepatic metastasis. Serum gastrin levels decreased to less than $25 \mathrm{pg} / \mathrm{ml}$ after antrectomy. Evaluation with upper 


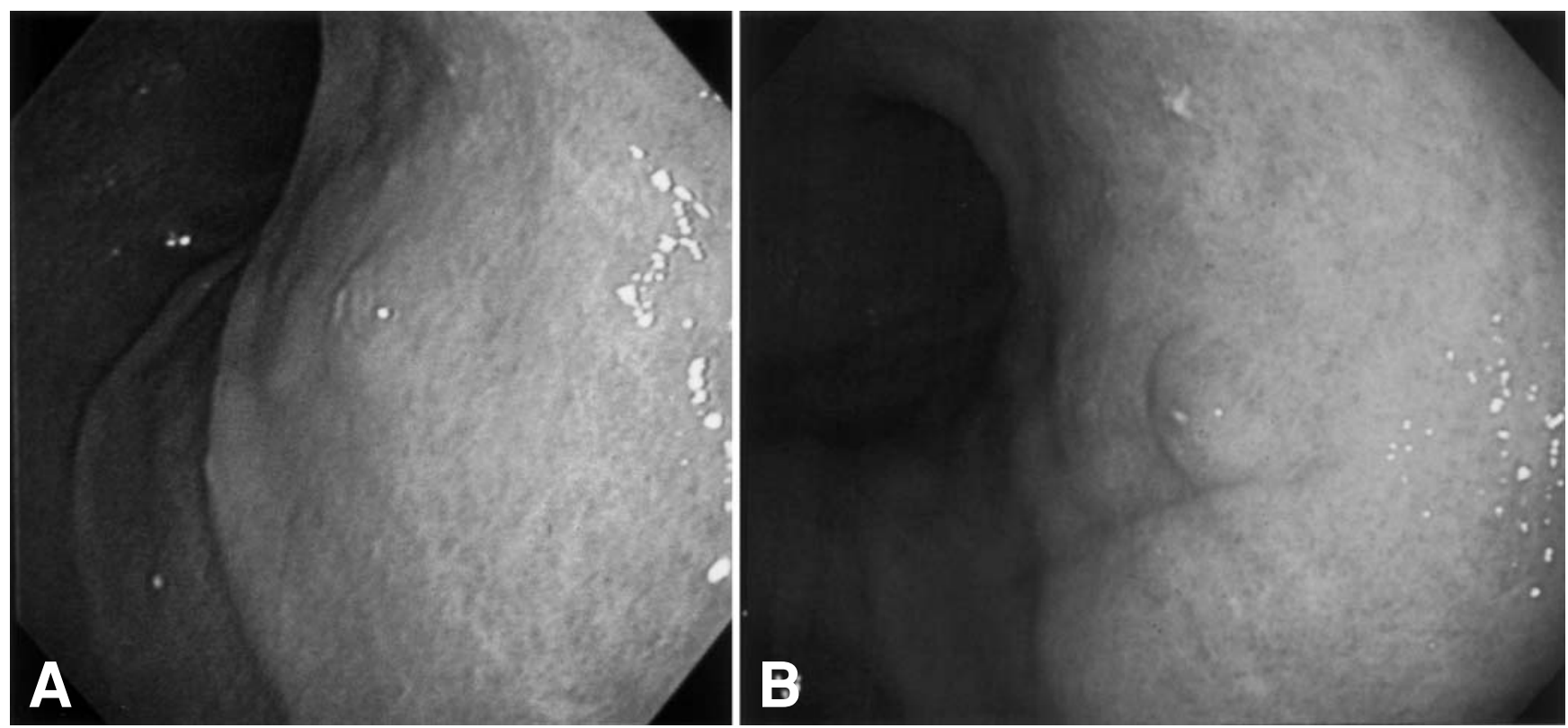

Fig. 1A,B. Case 2. A The initial endoscopic picture showed a polyp in the middle of the gastric body. Histologically, the biopsy specimens obtained from the polyp revealed carcinoid tumors. B The latest endoscopic picture (8 years after diagnosis) showed the polyps to have the same appearance and size as on the initial picture
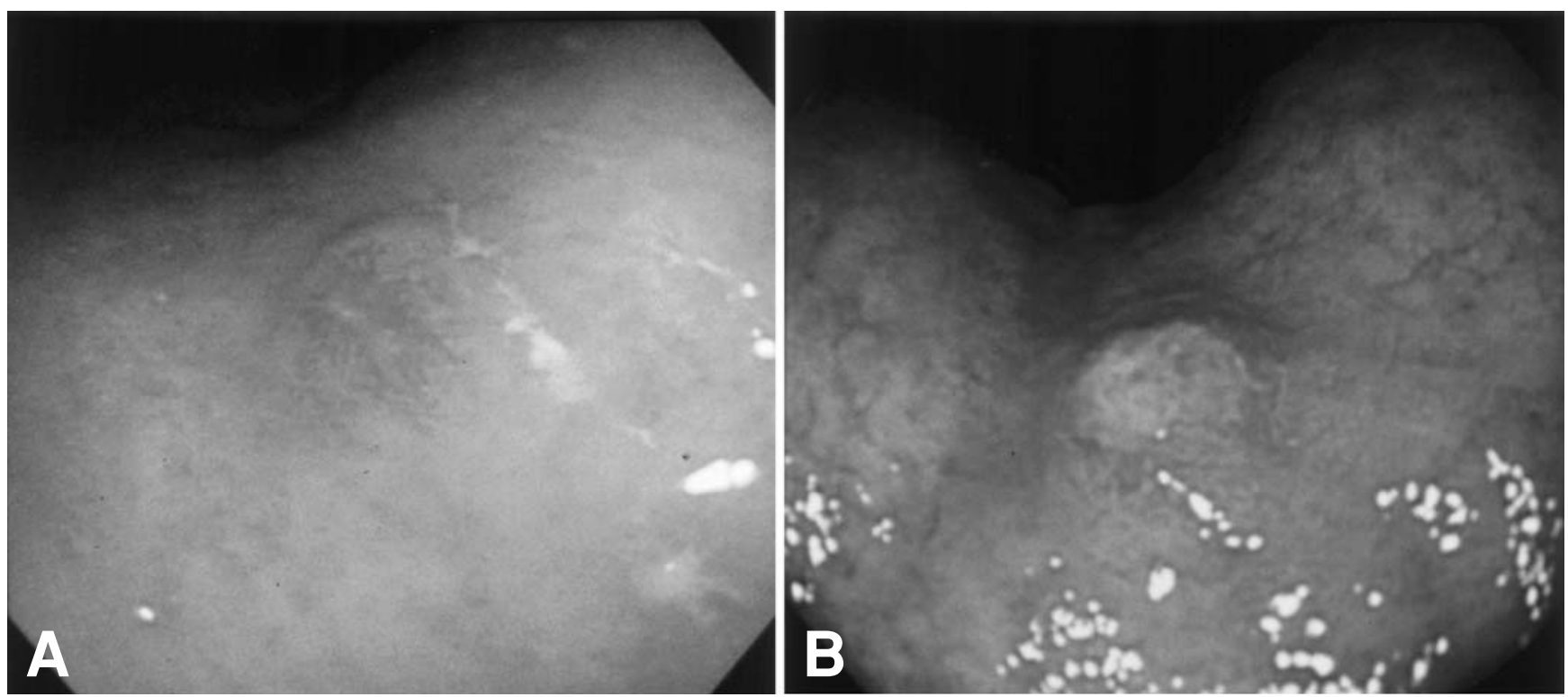

Fig. 2A,B. Case 4. A The initial endoscopic picture showed a polyp in the middle of the gastric body. Histologically, the biopsy specimens obtained from the polyp revealed carcinoid tumors. B The latest endoscopic picture (5 years after diagnosis) showed the polyps to have the same appearance and size as on the initial picture

gastrointestinal endoscopy and biopsy examination 4 to 6 months after antrectomy showed complete regression of disease in one patient and residual neoplasm in one patient, despite this patient's normal serum gastrin levels. Hirschowitz et al. [8] maintained that antrectomy may be the most appropriate treatment for this condi- tion, rather than total gastrectomy. Antrectomy in three patients resulted in normalization of serum gastrin levels within $8 \mathrm{~h}$ and the disappearance of carcinoids in 616 weeks, and follow up 21-30 months after antrectomy showed no carcinoids or enterochromaffin-like (ECL) cell hyperplasia. They concluded that multicentric ECL 

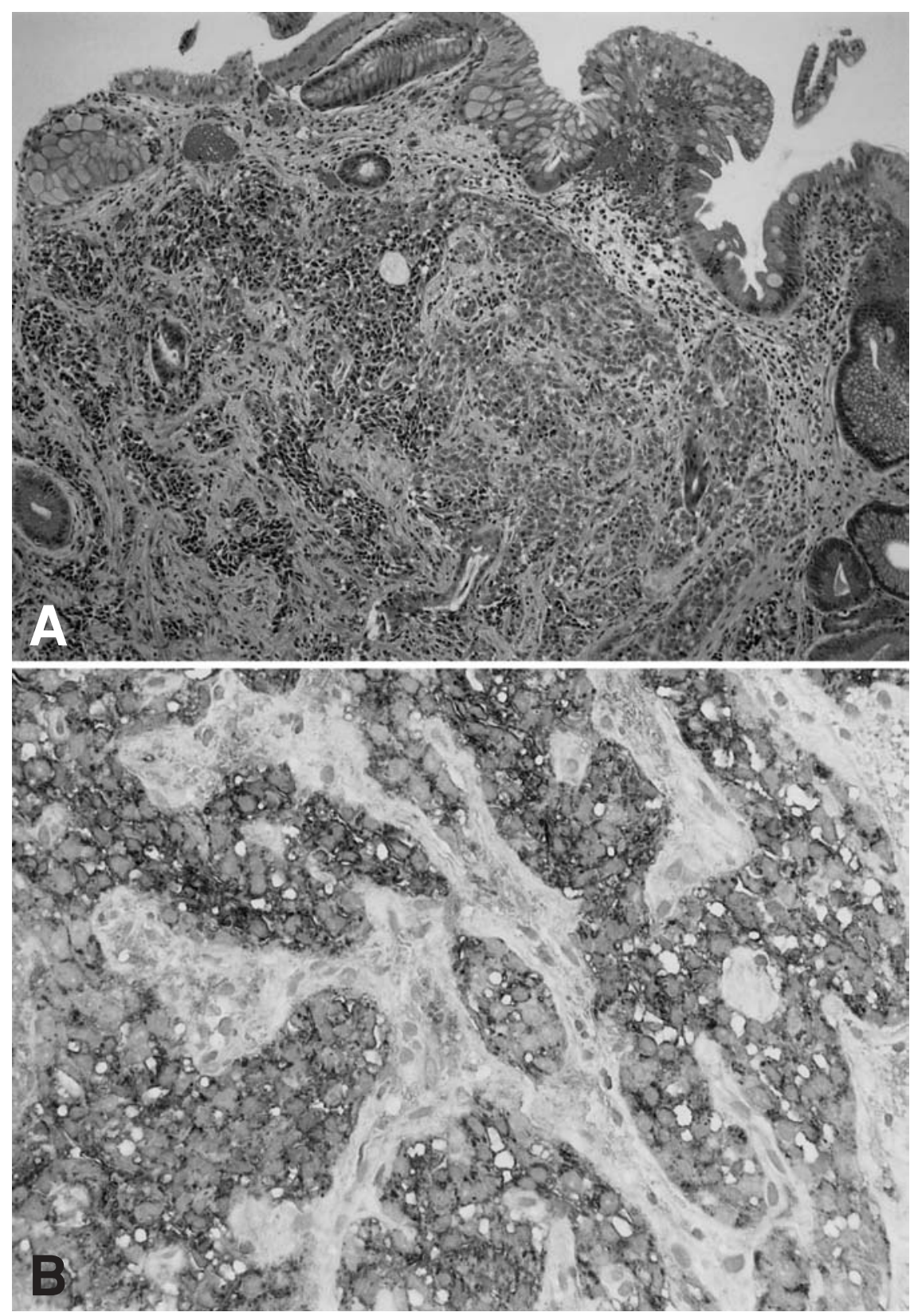

Fig. 3A,B. Case 1. A Histological examination of biopsy specimens obtained from the polyps 10 years after diagnosis revealed carcinoid tumor remaining in the mucosa. B The tumor cells showed immunoreactivity for chromogranin A. A $\mathrm{H} \& \mathrm{E}, \times 400 ; \mathbf{B} \times 400$ cell gastric carcinoids in patients with pernicious anemia and achlorhydria appeared to be gastrin-dependent and disappeared after the normalization of serum gastrin by antrectomy. Higham et al. [9] have suggested that an octreotide suppression test, coupled with the assay of histidine decarboxylase or chromogranin A gene expression, is useful in the assessment of gastric carcinoid tumors. They infused octreotide before antrectomy to suppress ECL cell and gastrin cell function. The infusion decreased plasma gastrin and gastrin synthesis. Moreover, there were marked reductions in markers of ECL cell function, e.g., histidine decarboxylase and chromogranin A messenger RNA abundance, in carcinoid tumor tissue, and the corpus mucosa was macroscopically normal. One year after antrectomy, the remaining stomach was normal.

In 1993, Rindi et al. [10] classified gastric carcinoid tumor into three subtypes, based on pathogenesis: (1) non-metastatic, arising in a background of body-fundus atrophic gastritis and hypergastrinemia; (2) locally metastatic, associated with hypertrophic gastropathy and hypergastrinemia due to multiple endocrine neoplasia/Zollinger-Ellison syndrome; and (3) sporadic cases. They suggested that gastrin-promoted carcinoids 
represented a benign or low-grade tumor disease, whereas sporadic carcinoids and neuroendocrine carcinomas were life-threatening neoplasms, independent of gastrin promotion [11]. Ahlman et al. [12] have suggested that a prospective treatment protocol for multiple gastric carcinoids with type A gastritis should be endoscopic removal of less numerous, small lesions as first-step therapy, followed by antrectomy at recurrence, while larger lesions should be excised in combination with antrectomy. Total gastrectomy is reserved for the rare cases of invasive tumors with lymph node metastases. Gilligan et al. [13] also maintained that hypergastrinemia-associated gastric carcinoids displayed relatively benign biological behavior, and that sporadic lesions required aggressive surgical management on diagnosis. They suggest that hypergastrinemiaassociated lesions can be managed initially by endoscopic excision of accessible tumors, followed by endoscopic follow up. If tumors recur, antrectomy and local excision may be used to remove the source of gastrin, resulting in cure in the vast majority of patients.

A few reports have described the endoscopic follow up of multiple gastric carcinoids associated with type A gastritis. Hori et al. [14] reported a patient who showed neither new lesions nor recurrence despite having persistent hypergastrinemia for 12 years after endoscopic mucosal resection for gastric carcinoid tumor. Rappel et al. [15] reported that the age-corrected Kaplan-Meier survival rate revealed normal life expectancy in 87 patients with multiple carcinoid tumors associated with type A gastritis; in $98 \%$ of these patients, the tumors had not been removed.

In 1993, we prospectively started a follow-up program for patients having multiple gastric carcinoids associated with type A gastritis. As stated above, the program consisted of endoscopic examinations at 6-month intervals and CT scans and blood tests, at 12-month intervals. The patients were to be immediately operated on if progression of the disease was discovered. From 1993 to 2003, eight patients were enrolled in this program. One patient was followed up for more than 10 years, and endoscopic examinations showed that the tumors had the same appearance and size as they did 10 years previously; CT showed no metastasis in spite of the patient's hypergastrinemia. In follow-up periods of 1 to 8 years in the other seven patients the lesions had not increased endoscopically, nor has there been distant metastasis.

We consider that multiple gastric carcinoids associated with type A gastritis are indolent, despite patients having continuous hypergastrinemia, and patients having such disease can be followed up with periodic endoscopic examination if their tumors are smaller than $2 \mathrm{~cm}$.

\section{References}

1. Strickland RG, Mackey IR. A reappraisal of the nature and significance of chronic atrophic gastritis. Am J Dig Dis 1973;18: 426-40.

2. Itsuno M, Watanabe $\mathrm{H}$, Iwafuchi M, Ito S, Yanaihara N, Sato K, et al. Multiple carcinoids and endocrine cell micronests in type A gastritis. Their morphology, histogenesis, and natural history. Cancer 1989;63:881-90.

3. Yamamoto C, Aoyagi K, Hizawa K, Ayabe S, Ariyama J, Sato H, et al. A case of multiple gastric carcinoid associated with type A gastritis (in Japanese with English abstract). Gastroenterol Endosc 1997;39:659-64.

4. Hosokawa O, Kaizaki Y, Watanabe K, Hattori M, Douden K, Hayashi $\mathrm{H}$, et al. Endoscopic surveillance of gastric carcinoid tumors associated with gastritis type A (in Japanese with English abstract). Stomach and Intestine 2000;35:1395-404.

5. Kaizaki Y, Hosokawa O, Fujii T, Nakaya T, Saito K. Histogenesis and changes of gastric neuroendocrine tumors - including MEN1 gene alteration (in Japanese with English abstract). Stomach and Intestine 2000;35:1355-64.

6. Aoyagi K, Koufuji K, Yano S, Murakami N, Terasaki Y, Yamasaki Y, et al. Multiple carcinoids in the stomach with hypergastrinemia and type A gastritis: a case report. Kurume Med J 2000;47:329-31.

7. Eckhauser FE, Lloyd RV, Thompson NW, Raper SE, Vinik AI. Antrectomy for multicentric, argyrophil gastric carcinoid: a preliminary report. Surgery 1988;104:1046-53.

8. Hirschowitz BI, Griffith J, Pellegrin D, Cummings OW. Rapid regression of enterochromaffinlike cell gastric carcinoids in pernicious anemia after antrectomy. Gastroenterology 1992;102:140918.

9. Higham AD, Dimaline R, Varro A, Attwood S, Armstrong G, Dockray GJ, et al. Octreotide suppression test predicts beneficial outcome from antrectomy in a patient with gastric carcinoid tumor. Gastroenterology 1998;114:817-22.

10. Rindi G, Luinetti O, Cornaggia M, Capella C, Solcia E. Three subtypes of gastric argyrophil carcinoid and gastric neuroendocrine carcinoma: a clinicopathologic study. Gastroenterology 1993;104:994-1006.

11. Sugamura K, Otani Y, Furukawa T, Saikawa Y, Yoshida M, Kubota $T$, et al. Gastric carcinoid tumors with aggressive lymphovascular invasion and lymph node metastasis. Gastric Cancer 2000;6:255-61.

12. Ahlman H, Kolby L, Lundell L, Olbe L, Wangberg B, Granerus $\mathrm{G}$, et al. Clinical management of gastric carcinoid tumors. Digestion 1994;55:77-85.

13. Gilligan CJ, Lawton GP, Tang LH, West AB, Modlin IM. Gastric carcinoid tumors: the biology and therapy of an enigmatic and controversial lesion. Am J Gastroenterol 1995;90:338-52.

14. Hori K, Fukui H, Imura J, Kojima T, Fujita M, Kawamata H, et al. Benign gastric carcinoid tumor with hypergastrinemia followed up for 12 years. Gastric Cancer 2000;3:161-4.

15. Rappel S, Altendorf-Hofmann, Stolte M. Prognosis of gastric carcinoid tumours. Digestion 1995;56:455-62. 